\title{
Unraveling the 'TGF- $\beta$ paradox' one metastamir at a time
}

\author{
Danny R Welch*1 and Douglas R Hurst ${ }^{2}$
}

\begin{abstract}
Transforming growth factor beta (TGF- $\beta$ ) has received noteworthy attention in the recent past due to its unique characteristic of functionally switching roles from tumor suppressor to metastasis promoter. To uncover the black box surrounding the mechanisms of TGF- $\beta$, Taylor and colleagues performed global miRNA expression analyses using a murine mammary carcinoma progression model. They discovered multiple miRNA regulated by TGF- $\beta$ and matrix stiffness. Focusing on miR-181a, they uncovered an intricate pathway regulating breast cancer metastasis that sheds new insight into metastasis regulation that may prove useful in clinical settings.
\end{abstract}

One of the greatest disappointments in the war on cancer is that survival for patients with metastasis remains abysmal (much less than 25\%), highlighting the dire need for improved treatment options. Metastasis is inexplicably complex, requires coordinated expression of multiple genes and proteins, and requires intricate communication of tumor cells to the constantly-changing environments as cells disseminate and colonize other tissues [1]. While the process is fortuitously inefficient, when cells succeed there are few effective treatment options. In addition to improving our clinical trial system [2], attention cannot be drawn away from the basic research that will unravel the cellular networks that drive metastasis.

Metastasis research has evolved in the past two decades. Early studies focused on individual genes or proteins that contribute to or inhibit the process. In the past few years, those individual metastasis-associated molecules are, not surprisingly, being assembled into coordinated pathways. Also, while researchers have long

*Correspondence: dwelch@kumc.edu

'Department of Cancer Biology, The Kansas University Medical Center, 3901 Rainbow Blvd, Wahl Hall West 2003, Mailstop 1071, Kansas City, KS 66160, USA Full list of author information is available at the end of the article recognized that metastatic cells interact with other cells, matrices and soluble molecules, roles of the microenvironment are becoming increasingly appreciated. Among myriad signaling molecules contributing to metastatic behavior is transforming growth factor beta (TGF- $\beta$ ).

A long-time, unsolved mystery in cancer biology is the paradoxical effect of TGF- $\beta$, which suppresses growth and tumorigenicity when cells are normal or near normal but somehow becomes a promoter of invasion and metastasis as neoplasms progress [3]. Both cell-autonomous and noncell-autonomous mechanisms have previously been invoked. In a recent publication by Taylor and colleagues [4], however, a new intermediary in the process has been uncovered. The results also highlight how microenvironment conditions, in this case matrix, are not inert bystanders in cellular behavior; and how the authors began the process of sorting through TGF- $\beta$ miRNA pathways that differentially mediate growth and invasive behaviors.

Launching from Weaver and colleagues' findings showing that tumor cells respond to matrix stiffness differentially [5] and that matrix rigidity - that often accompanies desmoplasia - differentially regulates TGF- $\beta$ responses, the Schiemann group grew breast carcinoma cells in two different matrices with a goal to mimic matrix tensegrity at primary tumors and metastatic sites [6]. Using threedimensional cultures of mammary carcinoma cells treated with TGF- $\beta$ in stiff or fractile matrices (Cultrex \pm type I collagen, respectively), differential responses were observed as expected. Global miRNA expression analyses were performed and a panel of metastasis-associated miRNA, so-called metastamir [7], was identified. They smartly focused on one miRNA, miR-181a, that was regulated in both tissue culture conditions and in three isogenic murine mammary carcinoma cell lines. The remainder of their studies characterized the role of miR$181 \mathrm{a}$ in promoting mammary cancer progression. Inhibition of miR-181a clearly altered lung colonization after tail vein injection. However, tumor latency, growth and dissemination did not appear to change (Note: the inhibition was lost in the tumor cells that successfully colonized, suggesting that they were revertants.) These 
findings further highlight how metastasis is a distinct phenotype from primary tumor growth.

As various laboratories dissect molecular mechanisms of metastatic spread, the identification of signaling pathways, including networks connecting matrices, signaling molecules and miRNA, is emerging. miRNA are increasingly recognized as key regulators of networks controlling normal cell functions and pathologies [8]. Progress has been slowed somewhat in defining miRNA since each miRNA can have as few as one or as many as hundreds of targets. Superimposing the intricate cellular interactions occurring throughout the metastatic process and the interconnectivity of miRNA in various signaling cascades, the complexity multiplies. Nonetheless, several themes begin to emerge.

We $[9,10]$ and others $[11,12]$ previously showed a metastasis suppressor-metastamir pathway; others have linked TGF- $\beta$ signaling with miRNA that regulate epithelialmesenchymal transition [13]; and, still others link miRNA as feedback to traditional signaling pathways [14]. As these networks become more finely elucidated, they are beginning to define metastasis mechanisms and patterns for particular molecular subtypes of cancer. Invoking miRNA represents an exciting avenue for translational studies since miRNA have taken centerstage in recent years as both biomarkers and directed therapy against cancer [15]. The miR-181a expression inversely correlated with patient survival, serving as a promising biomarker or therapeutic target. However, we urge caution with imposing findings on cancer subtypes at this time, since more extensively powered studies will be required to make firm such assertions.

While there is substantial promise, questions remain. How does matrix (composition or tensegrity) regulate TGF- $\beta$ signaling? Do all of the miRNA changed by TGF- $\beta$ work in concert? Are miRNA networks dependent upon the microenvironment? Are there bypass pathways or is there redundancy in miRNA pathways? How many of the other miRNA regulated by TGF- $\beta$ also change growth or metastasis? Are there ways in which the microenvironment could be treated to induce the growth-suppressive TGF- $\beta$ effects? These (and many other) questions will not be addressed in a single study. But tackling the pathways systematically (that is, one molecule at a time) may uncover networks that will translate into relevant personalized treatments.

Abbreviations

miRNA, micro RNA; TGF- $\beta$, transforming growth factor beta.
Competing interests

The authors declare that they have no competing interests.

\section{Acknowledgements}

The work in the authors' laboratories is supported by the National Cancer Institute (CA134981), Susan G. Komen for the Cure (SAC11037) and the National Foundation for Cancer Research - Center for Metastasis Research to DRW, and by the American Cancer Society (RSG-11-259-01-CSM) to DRH.

\section{Author details}

'Department of Cancer Biology, The Kansas University Medical Center, 3901 Rainbow Blvd, Wahl Hall West 2003, Mailstop 1071, Kansas City, KS 66160, USA. ${ }^{2}$ Department of Pathology, University of Alabama at Birmingham, 1670 University Blvd, G019 Volker Hall, Birmingham, AL 35294, USA.

Published: 27 February 2013

\section{References}

1. Hurst DR, Welch DR: Metastasis suppressor genes: at the interface between the environment and tumor cell growth. Int Rev Cell Mol Biol 2011, 286:107-180.

2. Steeg PS: Perspective: the right trials. Nature 2012, 485:S58-S59.

3. Bierie B, Moses HL: TGF $\beta$ : the molecular Jekyll and Hyde of cancer. Nat Rev Cancer 2006, 6:506-520.

4. Taylor MA, Sossey-Alaoui K, Thompson CL, Danielpour D, Schiemann WP: TGF-beta upregulates miR-181a expression to promote breast cancer metastasis. J Clin Invest 2013, 123:150-163.

5. Butcher DT, Alliston T, Weaver VM: A tense situation: forcing tumour progression. Nat Rev Cancer 2009, 9:108-122

6. Taylor MA, Amin JD, Kirschmann DA, Schiemann WP: Lysyl oxidase contributes to mechanotransduction-mediated regulation of transforming growth factor-beta signaling in breast cancer cells. Neoplasia 2011, 13:406-418.

7. Hurst DR, Edmonds MD, Welch DR: Metastamir: the field of metastasisregulatory microRNA is spreading. Cancer Res 2009, 69:7495-7498.

8. Bandyopadhyay S, Mitra R, Maulik U, Zhang MQ: Development of the human cancer microRNA network. Silence 2010, 1:6.

9. Hurst DR, Edmonds MD, Scott GK, Benz CC, Vaidya KS, Welch DR: Breast cancer metastasis suppressor 1 BRMS1 up-regulates miR-146 that suppresses breast cancer metastasis. Cancer Res 2009, 69:1279-1283.

10. Edmonds MD, Hurst DR, Vaidya KS, Stafford LJ, Chen D, Welch DR: Breast cancer metastasis suppressor 1 (BRMS1) coordinately regulates metastasis-associated microRNA expression. Int J Cancer 2009, 125:1778-1785.

11. Ma L, Teruya-Feldstein J, Weinberg RA: Tumour invasion and metastasis initiated by microRNA-10b in breast cancer. Nature 2007, 449:682-688.

12. Tavazoie SF, Alarcon C, Oskarsson T, Padua D, Wang QQ, Bos PD, Gerald WL, Massague J: Endogenous human microRNAs that suppress breast cancer metastasis. Nature 2008, 451:147-152.

13. Heldin CH, Lanstrom M, Moustakas A: Mechanism of TGF-beta signaling to growth arrest, apoptosis, and epithelial-mesenchymal transition. Curr Opin Cell Biol 2009, 21:166-176.

14. Ichimura A, Ruike Y, Terasawa K, Tsujimoto G: miRNAs and regulation of cell signaling. FEBS J 2011, 278:1610-1618.

15. Nana-Sinkam SP, Croce CM: Clinical applications for microRNAs in cancer Clin Pharmacol Ther 2013, 93:98-104.

doi: $10.1186 /$ bcr3383

Cite this article as: Welch DR, Hurst DR: Unraveling the 'TGF- $\beta$ paradox' one metastamir at a time. Breast Cancer Research 2013, 15:305. 